\title{
AVERAGE ALIASING ERROR FOR GENERAL PREFILTERING BASED ON SHIFT INVARIANT SPACES
}

\author{
Wen Chen \\ Department of Electronic Engineering \\ Jiaotong University, Minhang, Shanghai, China \\ Email: wenchen@sjtu.edu.cn
}

\begin{abstract}
Recently, the conventional A/D conversion has been extended to the A/D conversions based on shift invariant spaces, in which, the prefiltering is performed by quasi-projections into shift invariant spaces and sampling is performed in the shift invariant spaces. This paper studies the accuracy of the extended prefiltering. As a contribution of this paper, a formula to exactly evaluate the average aliasing error is established. Furthermore, for a signal in Sobolev space, removing the restriction that the exponent of Sobolev space is greater than $1 / 2$ imposed by the previous authors, this paper finds that the average aliasing error decays at the rate as the exponent of the Sobolev space.
\end{abstract}

Index Terms - prefiltering, aliasing error, quasi-projection, shift invariant spaces.

\section{INTRODUCTION}

In digital signal processing (DSP) and digital communications, an analog signal is converted to a digital signal by an analog-to-digital (A/D) converter. An ideal A/D converter prefilters an analog signal of finite energy by an ideal lowpass filter as illustrated by

$$
\text { Input signal } \longrightarrow \text { Ideal lowpass filter } \longrightarrow \text { Prefiltered signal, }
$$

and produces a discrete time (DT) signal by passing the prefiltered signal through an ideal impulse train. The derived DT signal is then subjected to a quantization process. Since an ideal lowpass filter and an ideal impulse train are impossible to be realized in hardware, a conventional practical A/D converter prefilters an analog signal of finite energy by a non-ideal lowpass filter and passes the prefiltered signal through a non-ideal impulse train.

Recent research on A/D conversion based on shift invariant spaces reveals that the conventional prefiltering by a lowpass filter can be extended and formulated as the prefiltering by quasi-projections into shift invariant spaces [5] as illustrated by

$$
\text { Input signal } \longrightarrow \text { Quasi-projection } \longrightarrow \text { Prefiltered signal. }
$$

Such an extension of prefiltering is significant and useful because it establishes a theoretical framework for the conventional prefiltering and conversely provides the new methods to design a non-ideal A/D converter of high accuracy and low computational complexity [5].

Supported by NSF China \#60672067, by NSF Shanghai \#062R14041, by Shanghai-Canada NRC \#06SN07112, by Cultivation Fund of the Key Scientific and Technical Innovation Project, Ministry of Education of China \#706022, and by Program for New Century Excellent Talents in University (NCET-06-0386).
An important issue in prefiltering is the difference between the prefiltered signal and the original signal, which is referred to as the aliasing error. For an ideal prefiltering, the aliasing error can be easily derived using the Parserval's identity. However, the estimate of aliasing error for the prefiltering by a quasi-projection into shift invariant space involves complicated analysis tools in approximation theory. For a smooth enough signal, certain kind of investigation has been done $[3,2,10]$, which claims that the aliasing error decays with respect to the scale of the shift invariant space, at the order of the Strang-Fix condition that the generator satisfies. Since a practical signal is usually non-smooth, the estimate of aliasing error for a nonsmooth signal is desirable for theoretical completeness and practical broadness.

The signals considered in [3] are taken from the Sobolev space. Since the only criterion for a signal in Sobolev space is the proper decay of its spectrum, Sobolev space is regarded to be an appropriate signal space for practical signal processing. The exponent of Sobolev space in [3] is assumed to be greater than $1 / 2$, which results in the Hölder continuity of the signal in Sobolev space, and hence excludes the large class of discontinuous signals. In the recent work done in [5], the authors derived an estimate of the aliasing error for the Lipschitz continuous signals in the sense of square norm, which can cover some discontinuous signals. But Lipschitz continuity of a signal in the sense of square norm is inconvenient to verify in practical manipulation.

For the practical purpose of convenience and the theoretical purpose of generality, it is desirable to establish the estimate of aliasing error for the signals in Sobolev space, but remove the restriction on the exponent of the Sobelev space imposed in [3]. One of our objectives in this paper is to establish such an estimate. Removing the restriction on the exponent of Sobolev space, this paper derives a formula to exactly evaluate the average aliasing error; Furthermore, for a signal in Sobolev space, the average aliasing error is found to decay at the order as the exponent of the Soblev space. In addition, we have derived the coefficient of the decay rate, which has lower computational complexity than the previous ones $[3,5]$.

\section{PREFILTERING BY QUASI-PROJECTIONS INTO SHIFT INVARIANT SPACES}

In this section, we will set up the prefiltering by quasi-projections into shift invariant spaces and introduces the related symbols and concepts, such as shift invariant space, Sobolev space, aliasing error, Fourier transform (FT), signals of finite energy, bandlimited signals and the Dirac signal. 


\subsection{Signals of finite energy, Fourier transform, bandlimited sig-} nals and the Dirac signal

An analog signal $f$ is of finite energy if the square norm $\|f\|_{2}=$ $\left(\int_{\mathbb{R}}|f(t)|^{2} d t\right)^{1 / 2}<\infty$. We also denote by $L^{2}(\mathbb{R})$ the signal space of finite energy, that is, $L^{2}(\mathbb{R})=\left\{f:\|f\|_{2}<\infty\right\}$. $f$ is said to be bandlimited if $F(\omega)=0$ whenever $|\omega|>\sigma$ for some $\sigma>0$, where $F$ is the spectrum or Fourier transform (FT) of $f$ defined by $F(\omega)=\int_{\mathbb{R}} f(t) e^{-j 2 \pi \omega t} d t$. In this case, $f$ is called a $\sigma$-band signal. The continuous time (CT) Dirac signal $\delta$ is defined as $\delta(t)=0$ for $t \neq 0$ and $\int_{\mathbb{R}} \delta(t) d t=1$; The discrete time (DT) Dirac signal is defined as $\delta[0]=1$ and $\delta[\ell]=0$ for $\ell \neq 0$.

\subsection{Shift invariant spaces}

For a $\lambda \geq 1$, the (scaled) shift invariant space (SIS) $V_{\lambda}(\varphi)$ generated by the generator $\varphi \in L^{2}(\mathbb{R})$ is defined as $[2,9]$,

$$
V_{\lambda}(\varphi):=\left\{\sum_{\ell} c_{\ell} \varphi(\lambda \cdot-\ell): \sum_{\ell}\left|c_{\ell}\right|^{2}<\infty\right\} \subset L^{2}(\mathbb{R}),
$$

where $\lambda$, called the scale of the SIS $V_{\lambda}(\varphi)$, is understood to be bandwidth in prefiltering or sampling ratio in sampling. In this paper, we also assume that $\{\varphi(\lambda \cdot-\ell)\}_{\ell}$ is a Riesz basis of $V_{\lambda}(\varphi)$, that is, $a \leq G_{\varphi} \leq b$ almost everywhere for some positive constants $a$ and $b$ [2], where $G_{\varphi}=\sum_{\ell}|\Phi(\cdot+\ell)|^{2}$ is called the ratio of orthonormality for the Riesz basis $\{\varphi(\cdot-\ell)\} \ell$. taking $\operatorname{sinc} t=\frac{\sin \pi t}{\pi t}$, the SIS $V_{\lambda}$ (sinc) is exactly the $\lambda$-band signal space of finite energy.

\subsection{Prefiltering and aliasing error}

The conventional ideal prefiltering by an idea lowpass filter boils down to a quasi-projection $P_{\text {sinc }}^{\lambda}: L^{2}(\mathbb{R}) \rightarrow V_{\lambda}$ (sinc), defined by,

$$
P_{\sin c}^{\lambda}(f):=\lambda \sum_{\ell}\langle f, \operatorname{sinc}(\lambda \cdot-\ell)\rangle \operatorname{sinc}(\lambda \cdot-\ell), \quad \forall f \in L^{2}(\mathbb{R})
$$

where $\langle\cdot, \cdot\rangle$ is the inne r product in $L^{2}(\mathbb{R})$ defined by $\langle f, g\rangle=\int_{\mathbb{R}} f(t)$ $g(t) d t$. Hence the aliasing error is $e_{f}^{\lambda}=f-P_{\text {sinc }}^{\lambda}(f)$, which can be made arbitrarily small by increasing the scale $\lambda$ of the SIS $V_{\lambda}$ (sinc), i.e., the bandwidth of the lowpass filter (see equaton (1)). This observation is very essential to establishing the sampling theory in shift invariant spaces $[4,6,7,8,12,13]$, and the prefiltering theory based on shift invariant spaces $[3,5]$, that will be also addressed in this paper. By replacing the generator sinc by a general generator $\varphi$, one can consider prefiltering a signal by a quasi-projections into a SIS (see equaton (2)), that is, to project a signal of finite energy to a SIS $V_{\lambda}(\varphi)$ by a quasi-projection $P_{\varphi}^{\lambda}: L^{2}(\mathbb{R}) \rightarrow V_{\lambda}(\varphi)$ defined by

$$
P_{\varphi}^{\lambda}(f):=\lambda \sum_{\ell}\langle f, \varphi(\lambda \cdot-\ell)\rangle \varphi(\lambda \cdot-\ell), \quad \forall f \in L^{2}(\mathbb{R}) .
$$

Then the aliasing error is defined as $e_{f}^{\lambda}=f-P_{\varphi}^{\lambda}(f)$. For the simplicity of realization, we do not consider the dual or integral operator version of quasi-projection in this paper $[3,10]$.

Taking the small random delay of the initial input to a practical prefiltering system or the small random initial phase of a practical sampler into account, it is reasonable to measure some kind of average aliasing error over a small delay interval or a small sampling period. Assume that the random delay of the initial input or the random initial phase of the sampler is uniformly distributed. Then the average aliasing error $\bar{e}_{f}^{\lambda}$ is defined as

$$
\bar{e}_{f}^{\lambda}:=\left\{\lambda \int_{0}^{1 / \lambda}\left\|e_{f_{u}}^{\lambda}\right\|_{2}^{2} d u\right\}^{1 / 2},
$$

where the delayed signal $f_{u}$ is defined as $f_{u}=f(\cdot-u)$. Since the scale $\lambda$, the bandwidth of the prefilter or the sampling ratio of the sampling, is usually very large, the average aliasing error is very close to the mean square error (MSE) of the prefiltering, i.e., $\bar{e}_{f}^{\lambda} \approx$ $\left\|e_{f}^{\lambda}\right\|_{2}$ for $f \in L^{2}(\mathbb{R})$.

\subsection{Sobolev spaces}

Let $r$ be a positive real number. The Sobolev space $W^{r}$ consists of all measurable functions $f$ satisfying $\int_{\mathbb{R}}|\omega|^{2 r}|\hat{f}(\omega)|^{2} d \omega<\infty$. In line with the definition of regularity, we use the notation $\left\|f^{(r)}\right\|_{2}=$ $\left(\int_{\mathbb{R}}|\omega|^{2 r}|\hat{f}(\omega)|^{2} d \omega\right)^{1 / 2}$. Sobolev space is a broad and appropriate signal space for the practical signals since the spectrum of signal in a Sobolev space decays appropriately. For example, in the conventional practical sampling where the actual sampling rate is less than the Nyquist sampling rate, it is appropriate to take the signal into a Sobolev space if the spectrum of the signal decays appropriately.

\subsection{Strang-Fix Condition}

In this paper, we also consider the Strang-Fix condition which has been widely used in approximation by shift invariant spaces $[2,10$, 11]. A generator $\varphi$ is said to satisfy the $L$ th order Strang-Fix condition if its spectrum $\Phi$ satisfies $\Phi(0) \neq 0$ and $\Phi^{(\alpha)}(\ell)=0$ for all integer $\ell \neq 0$ and $\alpha=0, \ldots, L-1$. We also assume that $\sum_{\ell}|t+\ell|^{L}|\varphi(t+\ell)|<\infty$, so that $\Phi$ has the bounded derivatives up to $L$.

\section{AVERAGE ALIASING ERROR FOR PREFILTERING BY QUASI-PROJECTIONS INTO SHIFT INVARIANT SPACES}

In this section, we are going to evaluate the average aliasing error for prefiltering by a quasi-projection into a shift invariant space. Furthermore, we will use the derived formula to estimate the average aliasing error for a signal in Sobolev space. Finally a numerical example based on the symmetric B-splines is calculated to demonstrate the good performance of general prefiltering as compared to the conventional prefiltering.

\subsection{Average aliasing error}

In this subsection, we are going to evaluate the average aliasing error for a signal of finite energy prefiltered by a quasi-projection into shift invariant space. For a generator $\varphi$, we define the kernel function $K_{\varphi}$ using the spectrum $\Phi$ of $\varphi$ as $K_{\varphi}:=1-2|\Phi|^{2}+|\Phi|^{2} G_{\varphi}$. It is of interest to note that the kernel can be simplified to $K_{\varphi}=1-|\Phi|^{2}$, if $\varphi$ is orthonormal. In particular, $K_{\sin c}=1-\chi_{[-1 / 2,1 / 2]}$ for the ideal prefiltering. Using the kernel $K_{\varphi}$ and combining the tools from approximation theory and classical analysis, we obtain the following simple formula to exactly calculate the average aliasing error.

Theorem 1 If a signal $f$ of finite energy is prefiltered by the quasiprojection $P_{\varphi}^{\lambda}$ into the SIS $V_{\lambda}(\varphi)$, then the average aliasing error is $\bar{e}_{f}^{\lambda}=\left\{\int_{\mathbb{R}}|F(\omega)|^{2} K_{\varphi}\left(\frac{\omega}{\lambda}\right) d \omega\right\}^{1 / 2}$, where $F$ is the spectrum of $f$. 
Compared to what obtained in [3], Theorem 1 removes the restrictions $f \in W^{r}$ and $r>1 / 2$. Since a practical signal is usually not smooth or continuous, this result, because of its applicability to any signal of finite energy, establishes a more general framework for the extended prefiltering. Theorem 1 also indicates that one can exactly calculate the average aliasing error for the prefiltering by quasi-projections. For example, the average aliasing error $\bar{e}_{f}^{\lambda}=\left\{\int_{\lambda}^{\infty}\left[|F(\omega)|^{2}+|F(-\omega)|^{2}\right] d \omega\right\}^{1 / 2}$ for the ideal prefiltering $(\varphi=$ sinc), which coincides with the the conventional argument on this issue, and hence can be made arbitrarily small by increasing the bandwidth $\lambda$.

\subsection{Signals in Sobolev spaces}

For the ideal prefiltering, by the argument in the last sub-subsection, if the spectrum $F$ decays at some rate, say $f \in W^{r}$ for some $r>0$, then the average aliasing error satisfies

$$
\bar{\varepsilon}_{f}^{\lambda} \leq \lambda^{-r}\left\{\int_{\lambda}^{\infty}\left[\left|\omega^{r} F(\omega)\right|^{2}+\left|\omega^{r} F(-\omega)\right|^{2}\right] d \omega\right\}^{1 / 2} \leq \lambda^{-r}\left\|_{f}(r)\right\|_{2}
$$

In the same spirit, it is desirable to establish a similar estimate of the average aliasing error for the prefiltering by quasi-projections into shift invariant spaces if $f$ belongs to the Sobolev space $W^{r}$ for some $r>0$. This is expressed in the following theorem.

Theorem 2 Assume that the kernel satisfies $\left\|K_{\varphi}|\cdot|^{-2 r}\right\|_{\infty}<\infty$. Then the average aliasing error satisfies $\bar{e}_{f}^{\lambda} \leq \lambda^{-r}\left\|f^{(r)}\right\|_{2} \| K_{\varphi}$ $|\cdot|^{-2 r} \|_{\infty}$, if a signal $f$ in the Sobolev space $W^{r}$ for some $r>0$ is prefiltered by the quasi-projection $P_{\varphi}^{\lambda}$.

Therefore the average aliasing error decays at the rate as the exponent of the Sobolev space. Compared to the error estimate for ideal prefiltering, there are additional multiplier $\left\|K_{\varphi}|\cdot|^{-2 r}\right\|_{\infty}$ in the average aliasing error for the general prefiltering. For $\varphi=$ sinc, the additional multiplier term $\left\|K_{\sin c}|\cdot|^{-2 r}\right\|_{\infty}=\|\left(1-\chi_{[-\lambda, \lambda]}\right) \mid$. $\left.\right|^{-2 r} \|_{\infty}=1$, which shows that this estimate covers the conventional ideal prefiltering.

The next step is to find the conditions on the generator $\varphi$ such that $\left\|K_{\varphi}|\cdot|^{-2 r}\right\|_{\infty}<\infty$. It is worth of indicating that a necessary condition for $\left\|K_{\varphi}|\cdot|^{-r}\right\|_{\infty} \leq \infty$ is $K(0)=0$, i.e., $G_{\varphi}(0)=$ $2-|\Phi(0)|^{-2}$. Since $G_{\varphi}(0)>0$, it deduces that $|\Phi(0)|>\sqrt{2} / 2$. Technically, it is easy to choose a generator such that $\Phi(0)=1$ by normalizing it as $\varphi / \Phi(0)$. Without loss of generality, we can assume that $\Phi(0)=1$ for a general generator. Combining with the necessary condition $K(0)=0$, we have $G_{\varphi}(0)=1$. Then $\Phi(\ell)=\delta[\ell]$ for any integer $\ell$, which exactly implies that $\varphi$ satisfies the first order Strang-Fix condition. Conversely, $\Phi(\ell+\omega)=\delta[\ell]+O(\omega)$ if $\varphi$ satisfies the first order Strang-Fix condition. Then $K_{\varphi}=O\left(\omega^{2}\right)$ by writing $K_{\varphi}=\left.\left.|1-| \Phi\right|^{2}\right|^{2}+|\Phi|^{2} \sum_{\ell \neq 0}|\Phi(\ell+\cdot)|^{2}$, and hence $\left\|K_{\varphi}|\cdot|^{-2 r}\right\|_{\infty}<\infty$ for $0<r \leq 1$. This is showed the following Corollary.

Corollary 1 Suppose that $\Phi(0)=1$. Then the generator $\varphi$ satisfies the first order Strang-Fix condition if and only if $\left\|K_{\varphi}|\cdot|^{-2 r}\right\|_{\infty}<\infty$ for some positive number $r \leq 1$. Consequently, the average aliasing error satisfies $\bar{e}_{f}^{\lambda} \leq \lambda^{-r}\left\|f^{(r)}\right\|_{2}\left\|K_{\varphi}|\cdot|^{-2 r}\right\|_{\infty}$, if a signal $f$ in the

Sobolev space $W^{r}$ is prefiltered by the quasi-projection $P_{\varphi}^{\lambda}$.

In general, if $\varphi$ satisfies the $L$ th order Strang-Fix condition for some positive integer $L$, we have the following criterion.
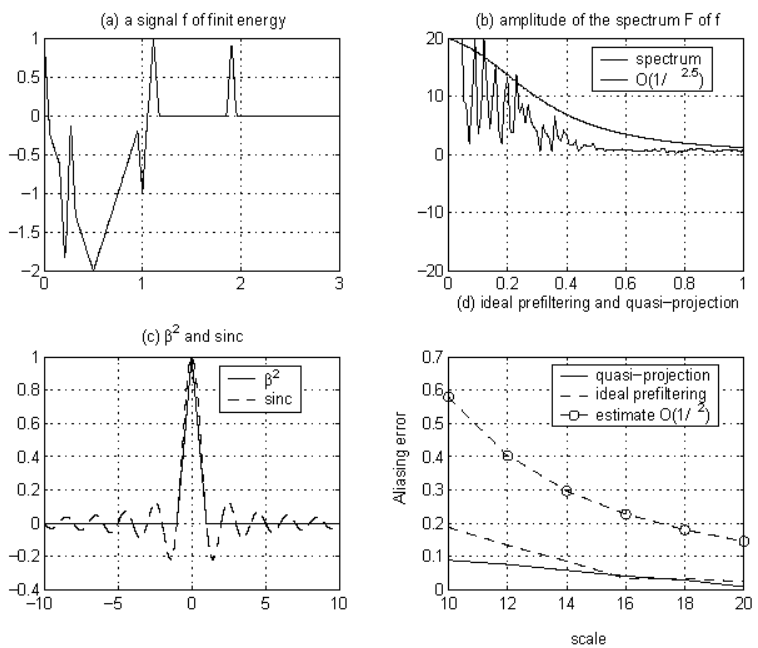

Fig. 1. (a) the input signal $f$; (b) The amplitude of the spectrum $F$ of the input signal $f$, which empirically decays at the rate $O\left(\omega^{-2.5}\right)$; (c) the B-spline of degree $2, \beta^{2}$, and the sinc function; (d) the aliasing errors for prefiltering by ideal lowpass filter of bandwidth $\lambda$ and by a quasi-projection $p_{\beta^{2}}^{\lambda}$ for the scale $\lambda=10 \ldots 20$.

Corollary 2 Assume that the generator $\varphi$ satisfies the Lth order Strang-Fix condition. Then there is a $\psi$, a finite linear combination of the shifts of $\varphi$, such that $\left\|K_{\psi}|\cdot|^{-2 r}\right\|_{\infty}<\infty$. Consequently, the average aliasing error $e_{f}^{\lambda}$ satisfies $\bar{e}_{f}^{\lambda} \leq \lambda^{-r}\left\|f^{(r)}\right\|_{2}|| K_{\psi}|\cdot|^{-2 r} \|_{\infty}$, if a signal $f$ in the Sobolev space $W^{r}$ for some $r>0$ is prefiltered by the quasi-projection $P_{\psi}^{\lambda}$ into $V_{\lambda}(\varphi)$ and $r \leq L$.

Intuitively it seems that Strang-Fix condition is too strong for obtaining the decay rate of the average aliasing error. But in some sense, the Strang-Fix condition is a necessary condition for the average aliasing error to decay at some rate $[3,2,10]$.

\subsection{Numerical results}

We give a numerical example to demonstrate the prefiltering based on the symmetric B-spline shift invariant space. A symmetric Bspline $\beta^{N}$ of degree $N$ is defined as the $N$-times convolution of the characteristic function $\chi_{[-1 / 2,1 / 2]}$, i.e., $\beta^{N}:=\chi_{[-1 / 2,1 / 2]} * \cdots *$ $\chi_{[-1 / 2,1 / 2]}$. Then Fourier transform of $\beta^{N}$ is sinc ${ }^{N}$. Since $K_{\beta^{N}}=$ $1-2 \operatorname{sinc}^{2 N}+\operatorname{sinc}^{2 N} G_{\beta^{N}}$, by Theorem 1, the average aliasing error is

$\bar{e}_{f}^{\lambda}=\int_{\mathbb{R}}|F(\omega)|^{2}\left[1-2 \operatorname{sinc}^{2 N}\left(\frac{\omega}{\lambda}\right)+\operatorname{sinc}^{2 N}\left(\frac{\omega}{\lambda}\right) G_{\beta^{N}}\left(\frac{\omega}{\lambda}\right)\right] d \omega$

, if a signal $f$ of finite energy is prefiltered by the quasi-projection $P_{\beta^{N}}^{\lambda}$ into the SIS $V_{\lambda}\left(\beta^{N}\right)$.

Suppose that a signal $f$ is taken from the Sobolev space $W^{r}$ for some $r>0$, and prefiltered by the quasi-projection $P_{\beta^{N}}^{\lambda}$. Since Matlab shows that $\left\|K_{\beta^{N}}|\cdot|^{-4}\right\|_{\infty} \leq 11 N^{2}$, by Theorem 2, the average aliasing error satisfies $\bar{e}_{f}^{\lambda} \leq 11 N^{2} \lambda^{-r}\left\|f^{(r)}\right\|_{2}$, if $r \leq$ 2. Noting that the $\beta^{N}$ satisfies the $N$ th order Strang-Fix condition, by Corollary 2 , there is $\psi^{N}$, a finite linear combination of the shifts of $\beta^{N}$, such that the average aliasing error satisfies $\bar{e}_{f}^{\lambda} \leq$ $\lambda^{-r}\left\|f^{(r)}\right\|_{2}\left\|K_{\psi^{N}}|\cdot|^{-2 r}\right\|_{\infty}$, if $f$ is prefiltered by $P_{\psi}^{\lambda}$ and $r \leq$ 


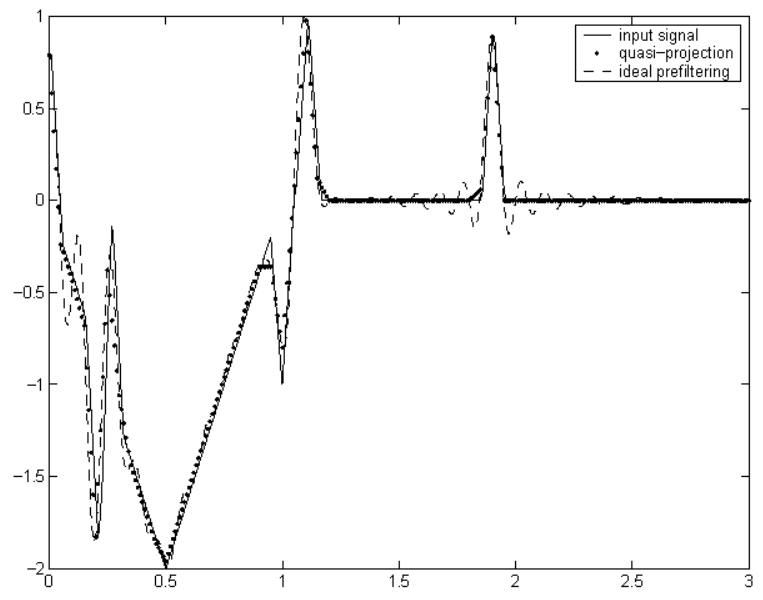

Fig. 2. The original input signal $f$, the prefiltered signal by quasiprojection $P_{\beta^{2}}^{\lambda}$ and the ideal prefiltering $P_{\text {sinc }}^{\lambda}$. The ideally prefiltered signal $P_{\mathrm{sinc}}^{\lambda}(f)$ introduces ripples in the smooth part of $f$; while the prefiltered signal $P_{\beta^{2}}^{\lambda}(f)$ approximates $f$ very well. In this simulation, $\lambda=20$.

$N$. In the following, we try to figure out the $\psi^{N}$ for the small $N$, e.g., $N=2,3$. Since $\left(\operatorname{sinc}^{2}\right)^{\prime}(0)=0$, we can take $\psi^{2}=\beta^{2}$. Since $\left(\operatorname{sinc}^{3}\right)^{\prime}(0)=0$ and $\left(\operatorname{sinc}^{3}\right)^{\prime \prime}=-1$, using $\Psi^{(\alpha)}(0)=$ $\sum_{k=0}^{\alpha}\left(\begin{array}{l}\alpha \\ k\end{array}\right) P_{2}^{(\alpha-k)}(0)\left(\operatorname{sinc}^{2}\right)^{(k)}(0)=\delta[\alpha]$ for $\alpha=0,1,2$, we have $P_{2}(0)=1, P_{2}^{\prime}(0)=0, P_{2}^{\prime \prime}(0)=1$. This solves $c_{0}=\frac{8 \pi^{2}-1}{8 \pi^{2}}$ $c_{1}=\frac{-1}{8 \pi^{2}}$ and $c_{2}=\frac{1}{4 \pi^{2}}$. Therefore $\psi^{3}=\frac{8 \pi^{2}-1}{8 \pi^{2}} \varphi-\frac{1}{8 \pi^{2}} \varphi(\cdot-1)+$ $\frac{1}{4 \pi^{2}} \varphi(\cdot-2)$. We leave the interesting calculation for $N>3$ to the interested readers. We now turn to consider prefiltering a physical signal based on the symmetric B-spline of degree 2 and compare it with the ideal prefiltering.

Consider the input signal $f$ as shown in Fig. 1 (a). The spectrum $F$ of $f$ is shown in Fig. 1 (b). Empirical estimate shows that $F(\omega)=O\left(\omega^{-2.5}\right)$ at infinity (see Fig. $1(\mathrm{~b})$ ), and hence $f \in W^{r}$ for $r<2$. For simplicity, we use the prefiltering $P_{\beta^{2}}^{\lambda}$ (see Fig. 1 (c) for the graph of $\left.\beta^{2}\right)$. Since Matlab shows that $K_{\beta^{2}}(\omega) /|\omega|^{4} \leq 45$, the average aliasing error satisfies $\bar{e}_{f}^{\lambda} \leq 45\left\|f^{(r)}\right\|_{2} \lambda^{-r}$ for $r<2$. Fig. 1 (d) shows this estimate versus the actual aliasing error for $\lambda=10, \ldots, 20$. The actual aliasing error is seen to be lower than the estimate, which implies that our estimate is not yet optimal. We also put the actual aliasing error for the ideal lowpass prefiltering in Fig. 1 (d) for comparison. It is observed that the actual accuracy of prefiltering by $P_{\beta^{2}}^{\lambda}$ is superior to that by $P_{\text {sinc }}^{\lambda}$ even if their theoretical estimates are in the same order. Visually, we see that the prefiltering by a quasi-projection into $V_{\lambda}\left(\beta^{2}\right)$ provides good approximation. In Fig. 2, the ideal lowpass prefiltering $P_{\text {sinc }}^{\lambda}$ introduces ripples in the smooth part of $f$. However, $P_{\beta^{2}}^{\lambda}(f)$ approximates $f$ very well. Since $\beta^{2}$ is compactly supported, this means reduction of the computational complexity in prefiltering.

\section{CONCLUSIONS}

In this paper, we investigate the prefiltering by quasi-projections into SIS. This paper establishes a formula to exactly calculate the average aliasing error for a signal prefiltered by a quasi-projection. Furthermore, removing the restriction on the exponent of Soboleve space, this paper finds that the average aliasing error decays at the order as the exponent of the Sobolev space as long as the generator satisfies sufficient order Strang-Fix condition. Numerical results show that, compared to the ideal prefiltering, the prefiltering by quasiprojection offers high accuracy for some signals. It also reduces the computational complexity in prefiltering by choosing the generators of fast decay or compact support. Since a prefiltered signal can be perfectly reconstructed from the sampled DT signals by passing the DT signals through a proper admissible filter, and the admissible filters in time domain can be made to be of fast decay or compactly supported, it reduces the noise sensisivity and computational complexity in D/A conversion. Therefore, the extended A/D conversion offers rich choices to design a non-ideal A/D conversion system of high accuracy and low computational complexity in prefiltering, as well as low computational complexity and reduced noise sensitivity in D/A conversion. Meanwhile, such A/D conversion scheme based on shift invariant space is amenable to the arbitrary band signals by choosing a generator such that its spectrum matches the bands of the practical signals.

\section{REFERENCES}

[1] A. Aldroubi, and K. Gröchenig, "Non-uniform sampling and reconstruction in shift-invariant spaces," SIAM Review, vol. 43, no. 4, pp. 858-620, 2001.

[2] C. deBoor, C. deVore, and A. Ron, "Approximation from shift invariant subspaces of $L^{2}\left(\mathbb{R}^{d}\right)$," Trans. American Mathematical Society, vol. 341, pp. 787-806, 1994.

[3] T. Blu, and M. Unser, "Approximation error f Quasiinterpolators and (Multi-)wavelet expansions," Applied and Computational harmonic Analysis, vol. 6, pp. 219-251, 1999.

[4] W. Chen, B. Han, and R.-Q. Jia "On simple oversampled A/D conversion in shift invariant spaces," IEEE Trans. Information Theory, vol. 51, no. 2, 2005.

[5] W. Chen, B. Han, and R.-Q. Jia "Estimate of aliasing error for non-smooth signals prefiltered by quasi-projections into shift invariant spaces," IEEE Trans. Signal Processing, vol. 53, no. 5, pp. 1927-1933, 2005.

[6] W. Chen and S. Itoh, "A sampling theorem for shift invariant subspaces," IEEE Trans. Signal Processing, vol. 46, no. 10, pp. 2822-2824, 1998.

[7] W. Chen and S. Itoh, "Oversampling theorem for wavelet subspaces," IEICE Trans. Fundamentals, vol. 81, no. 1, pp. 131$138,1998$.

[8] W. Chen, S. Itoh, and J. Shiki "On sampling in shift invariant spaces," IEEE Trans. Information Theory, vol. 48, no. 10, pp. 2802-2810, 2002.

[9] R.-Q. Jia, "Shift-invariant spaces and linear operator equations," Israel Journal of Mathematics, vol. 103, pp. 259-288, 1998.

[10] J. Lei, R.-Q. Jia, and E. W. Cheney, "Approximation from shift invariant spaces by integral operators," SIAM Journal of Math. Analysis, vol. 28, no. 2, pp. 481-498, 1997.

[11] G. Strang, and G. Fix, "A Fourier analysis of finite element variational method" in Constructive Aspects of Functional Analysis (G. Geymonat Ed.) pp. 793-840, C. I. M. E., 1973.

[12] M. Unser, "Sampling — 50 years after Shannon," Proc. of the IEEE, vol. 88, no. 4, pp. 569-589, 2000.

[13] G. G. Walter, "A sampling theorem for wavelet subspaces," IEEE Trans. Information Theory, vol. 38, no. 2, pp. 881-884, 1992. 